\title{
CENTRALIZER NEAR-RINGS THAT ARE RINGS
}

\author{
JUTTA HAUSEN and JOHNNY A. JOHNSON
}

(Received 2 October 1992)

Communicated by P. Schultz

\begin{abstract}
Given an $R$-module $M$, the centralizer near-ring $\mathscr{M}_{R}(M)$ is the set of all functions $f: M \rightarrow M$ with $f(x r)=f(x) r$ for all $x \in M$ and $r \in R$ endowed with point-wise addition and composition of functions as multiplication. In general, $\mathscr{M}_{R}(M)$ is not a ring but is a near-ring containing the endomorphism ring $E_{R}(M)$ of $M$. Necessary and/or sufficient conditions are derived for $\mathscr{M}_{R}(M)$ to be a ring. For the case that $R$ is a Dedekind domain, the $R$-modules $M$ are characterized for which (i) $\mathscr{M}_{R}(M)$ is a ring; and (ii) $\mathscr{M}_{R}(M)=E_{R}(M)$. It is shown that over Dedekind domains with finite prime spectrum properties (i) and (ii) are equivalent.
\end{abstract}

1991 Mathematics subject classification (Amer. Math. Soc.): 16D70, 16S50, 16 Y30.

\section{Introduction}

Throughout, the word ring is used to mean an associative ring with identity and all modules will be unital right modules.

Given modules $M$ and $N$ over a ring $R$, an $R$-homogeneous map from $M$ to $N$ is a function $f: M \rightarrow N$ such that, for all $x \in M$ and for all $r \in R, f(x r)=f(x) r$. The set of all homogeneous maps from $M$ to $M$ is denoted by $\mathscr{M}_{R}(M)$, and $\mathscr{M}_{R}(M)$ is a zero-symmetric abelian near-ring with identity under the usual operations of pointwise addition and composition of functions [16]. This near-ring, sometimes called the centralizer near-ring determined by $R$ and $M$ [11-15], obviously contains the ring $E_{R}(M)$ of all $R$-endomorphisms of $M$ :

$$
E_{R}(M) \subseteq \mathscr{M}_{R}(M)
$$

and satisfies all the ring axioms with the possible exception of the left distributive law,

(C) 1995 Australian Mathematical Society $0263-6115 / 95 \$ A 2.00+0.00$ 
that is

(1.2) $\mathscr{M}_{R}(M)$ is a ring $\Longleftrightarrow \forall f, g, h \in \mathscr{M}_{R}(M), f(g+h)=f g+f h$.

In a recent paper [7], Fuchs, Maxson and Pilz have considered the class $\mathscr{R}$ of all rings $R$ such that, for every $R$-module $M, \mathscr{M}_{R}(M)$ is a ring. They prove that a ring $R$ belongs to $\mathscr{R}$ (if and) only if $\mathscr{M}_{R}(M)=E_{R}(M)$ for all $R$-modules $M$. The focus in [7] is on the rings in $\mathscr{R}$ and their structure. They show that every ring in $\mathscr{R}$ is non-commutative and must have non-zero zero divisors.

The current paper concerns itself with the structure of modules whose centralizer near-ring is a ring without imposing this condition on the entire module class. It will be convenient to call the $R$-module $M$ semi-endomorphal if $\mathscr{M}_{R}(M)$ is a ring. If $\mathscr{M}_{R}(M)=E_{R}(M)$, that is, if every $R$-homogeneous map from $M$ to $M$ is an endomorphism, then $M$ is said to be endomorphal.

We pose

PROBLEM 1.3. Given a ring $R$, find necessary and/or sufficient conditions for an $R$-module $M$ to be semi-endomorphal.

PROBLEM 1.4. Given a ring $R$, find necessary and/or sufficient conditions for an $R$-module $M$ to be endomorphal.

PROBLEM 1.5. Describe the rings $R$ over which there exist semi-endomorphal modules which are not endomorphal.

After general results for modules over arbitrary rings, we let $R$ be an integral domain. Given a torsion-free $R$-module $M$, we show that $\mathscr{M}_{R}(M)=E_{R}(M)$ if and only if $M$ is an $R$-submodule of the quotient field $Q$ of $R$; and $\mathscr{M}_{R}(M)$ is a ring if and only if any two $R$-independent elements of $M$ are of incomparable type. Modules of the latter kind are said to be absolutely anisotropic. For $R$ a Dedekind domain, we obtain a complete characterization: the $R$-module $M$ is semi-endomorphal if and only if either $R$ is a submodule of $Q / R$, or $M$ is torsion-free absolutely anisotropic. For $R$ a principal ideal domain, the endomorphal $R$-modules are shown to be precisely the locally cyclic modules; if $R$ has only finitely many prime ideals then every semiendomorphal $R$-module is endomorphal. However, there do exist semi-endomorphal $R$-modules which are not endomorphal if the prime spectrum of $R$ and $R$ have equal cardinality. Thus, there exist abelian groups $G$ such that $\mathscr{M}_{\mathbb{Z}}(G)$ is a ring but $\mathscr{M}_{\mathbb{Z}}(G) \neq E_{\mathbb{Z}}(G)$.

\section{Modules over arbritrary rings}

Let $R$ be any ring. The $R$-module $M$ is said to be locally cyclic if, given any two elements $x$ and $y$ in $M$, there exist $a \in M$ such that both $x$ and $y$ belong to $a R$. One 
easily verifies

PROPOSITION 2.1. Every locally cyclic module is endomorphal. In fact, if $X$ is locally cyclic and $f: X \rightarrow M$ is any $R$-homogeneous map, then $f \in \operatorname{Hom}_{R}(X, M)$.

Maxson and Smith give an example of a module which is semi-endomorphal but not endomorphal [13, Example 2.1]. One verifies that their example is of the form described in

PROPOSITION 2.2. If the $R$-module $M$ has a family $\left\{X_{i}\right\}_{i \in I}$ of submodules $X_{i}$ such that (i) $M=\bigcup_{i \in I} X_{i}$, and (ii) for each $f \in \mathscr{M}_{R}(M)$ and for each $i \in I, f \mid X_{i} \in$ $\operatorname{Hom}_{R}\left(X_{i}, X_{i}\right)$, then $\mathscr{M}_{R}(M)$ is a ring.

PROOF. It suffices to verify (1.2). Let $f, g, h \in \mathscr{M}_{R}(M)$ and let $a \in M$. Then $a \in X_{i}$ for some $i \in I$. By hypothesis, $g(a), h(a) \in X_{i}$ and $f \mid X_{i}$ is a homomorphism. Thus $f(g+h)(a)=f(g(a)+h(a))=f g(a)+f h(a)=(f g+f h)(a)$ as desired.

In order to derive necessary conditions for a module to be semi-endomorphal, the existence of non-trivial direct sum decompositions proves useful (cf. [7, 14]). One easily proves

LEMMA 2.3. Direct summands of (semi-)endomorphal modules are (semi-)endomorphal.

For ease of reference, we include the following result from [14].

LEMMA 2.4. ([14, 2.1]) Suppose that $M$ is an $R$-module and $A$ and $B$ are submodules of $M$ such that $M=A \oplus B$. If $M$ is semi-endomorphal and $f \in \mathscr{M}_{R}(M)$, then $f(a+b)=f(a)+f(b)$ for all $a \in A$ and all $b \in B$.

A general method for constructing $R$-homogeneous maps was given by Maxson and van der Walt in [14]. We modify their construction. Let $Y \subseteq M$ be a non-empty subset of the $R$-module $M$. If, for all $r \in R$ and all $y \in Y, y r \in Y$ then $Y$ is said to be $R$-closed; define $Y$ to be strongly $R$-pure if $a \in M, r \in R$ and $0 \neq a r \in Y$ imply $a \in Y$. Note that $Y$ is an $R$-closed and strongly $R$-pure subset of $M$ if and only if the set $X=Y \backslash\{0\}$ satisfies (ii) and (iii) of [14, 2.2].

LEMMA 2.5. Let $X$ be a subset of the $R$-module $M$ which is $R$-closed and strongly $R$ pure, and let $\varphi: X \rightarrow M$ be an $R$-homogeneous map. Define a function $f: M \rightarrow M$ by

$$
f(a)=\left\{\begin{array}{lc}
\varphi(a) & \text { if } a \in X, \\
0 & \text { otherwise. }
\end{array}\right.
$$

Then $f$ is an $R$-homogeneous map. 
Proof. Note that $f(0)=\varphi(0)=0$. Let $r \in R$ and $a \in M$. If $a \in X$, then $f(a r)=\varphi(a r)=\varphi(a) r=f(a) r$. Suppose $a \notin X$. Then $f(a)=0$ and either ar $\notin X$ or $a r=0$. In either case, $f(a r)=0=f(a) r$.

An $R$-module $M$ is said to be torsion-free if, for $a \in M$ and $r \in R$, ar $=0$ implies $a=0$ or $r=0$. Clearly, the existence of a non-zero torsion-free $R$-module implies that $R$ is a ring without zero-divisors.

REMARK 2.6. Let $M$ be an $R$-module.

(i) If $X$ is a submodule of $M$ such that the quotient module $G / X$ is torsion-free, then $X$ is an $R$-closed and strongly $R$-pure subset of $M$.

(ii) The union of any family of $R$-closed and strongly $R$-pure subsets of $M$ is $R$-closed and strongly $R$-pure.

Maxson and van der Walt show that semi-endomorphal modules are either indecomposable, or what they term $R$-connected $[14,2.3]$. We shall require the following result.

LEMMA 2.7. Let $M$ be a semi-endomorphal $R$-module which has a non-trivial direct sum decomposition. If $S$ is a submodule of $M$ such that $M / S$ is torsion-free, then $S=M$.

PROOF. It suffices to show that $S$ contains every proper direct summand of $M$. Let $M=A \oplus B$ with $B \neq 0$. Assume $A \nsubseteq S$. Then there is $a \in A \backslash S$. Put $X=(A \cap S) \oplus B$. Then $X$ is a submodule of $M$ with torsion-free quotient $M / X$. By 2.6 and 2.5, the map $f: M \rightarrow M$ defined by $f(x)=x$ if $x \in X$ and $f(x)=0$ otherwise is $R$-homogeneous. Let $0 \neq b \in B$. Then $b \in X$, and both $a$ and $a+b$ are not in $X$. Thus

$$
f(a+b)=0 \neq b=f(b)=f(a)+f(b),
$$

and $M$ is not semi-endomorphal, by 2.2 .

COROLLARY 2.8. Torsion-free semi-endomorphal modules are indecomposable.

\section{Modules over integral domains}

From now on we assume that $R$ is an integral domain. We use the terminology of [9]: if $x \in M$ is an element of an $R$-module $M$, the order ideal of $x$ is the set

$$
o(x)=\{r \in R \mid x r=0\},
$$


and $x$ is said to be a torsion element if its order ideal is non-zero; the set $T(M)$ of all torsion elements of $M$ is a submodule of $M$ such that the quotient module $M / T(M)$ is torsion-free. Applying 2.7 with $S=T(M)$ yields

LEMMA 3.1. Let $M$ be a module over an integral domain which has a non-trivial direct sum decomposition. If $M$ is semi-endomorphal then $M$ is a torsion module.

Throughout, we let $Q$ denote the quotient field of $R$. If $M$ is a torsion-free $R$-module then $M$ may be regarded as an $R$-submodule of the vector space $V=$ $M \otimes_{R} Q$. The rank of $M$ is defined to be the $Q$-dimension of $V$. The torsion-free rank-one $R$-modules are, up to isomorphism, precisely the non-zero $R$-submodules of $Q$.

An easy calculation shows that $Q$ is a locally cyclic $R$-module. Since quotient modules of locally cyclic modules are locally cyclic, 2.1 implies

LEMMA 3.2. The quotient field $Q$ of $R$ and the factor module $Q / R$ are locally cyclic endomorphal $R$-modules.

Submodules of locally cyclic modules need not be locally cyclic. However, we have

LEMMA 3.3. Every torsion-free rank-one module over an integral domain is endomorphal. In fact, if $X$ and $M$ are torsion-free $R$-modules with $X$ of rank one and $f: X \rightarrow M$ is an R-homogeneous map, then $f \in \operatorname{Hom}_{R}(X, M)$.

ProOF. Assume the hypotheses. Let $x$ and $y$ be non-zero elements of $X$. Then there exist non-zero $r, s \in R$ with $r x=s y$. Computation shows that $r s f(x+y)=$ $r s(f(x)+f(y))$.

Two torsion-free $R$-modules $M$ and $N$ are said to be quasi-isomorphic, in symbols $M \dot{\sim} N$, if each is isomorphic to a submodule of the other. Quasi-isomorphism is an equivalence relation on the class of torsion-free $R$-modules. For a torsion-free rank-one module $A$, the type of $A$ is the equivalence class containing $A$ :

$$
\mathbf{t}(A)=\{B \in \operatorname{Mod}-R \mid B \text { torsion-free and } B \dot{\check{\simeq}} A\} .
$$

Two types $\mathbf{t}(A)$ and $\mathrm{t}(B)$ are said to be incomparable if $\operatorname{Hom}_{R}(A, B)=0$ and $\operatorname{Hom}_{R}(B, A)=0$. Using the fact that $Q$ is the injective envelope of each of its non-zero $R$-submodules [18, p. 50], one verifies that this definition is independent of the choice of representatives in the two types. If $x$ is a non-zero element of the torsion-free $R$-module $M$, the pure submodule generated by $x$ is

$$
\langle x\rangle_{*}=\{y \in M \mid y r \in x R \text { for some non-zero } r \in R\},
$$


and the type of $x$ is defined to be the type of $\langle x\rangle_{*}: \mathbf{t}(x)=\mathbf{t}\left(\langle x\rangle_{*}\right)$. For abelian groups, of course, these notions agree with the standard definitions [6].

Beaumont and Pierce call a torsion-free abelain group $G$ completely anisotropic if no two independent elements of $G$ have the same type [2, p. 28]. We modify this notion by calling a torsion-free module over an integral domain absolutely anisotropic if any two independent elements have incomparable type. The existence of absolutely anisotropic torsion-free abelian groups of rank two was established in [2] (see also [1, $8])$. In our context we have

THEOREM 3.4. Let $M$ be a torsion-free module over an integral domain $R$. Then $\mathscr{M}_{R}(M)$ is a ring if and only if $M$ is absolutely anisotropic.

PROOF. Let $M$ be a torsion-free $R$-module. Suppose, firstly, that $M$ is semiendomorphal. By way of contradiction, assume $M$ contains two independent elements whose types are comparable. Let $X$ and $Y$ denote the pure rank-one submodules they generate. Without loss of generality, we may assume $\operatorname{Hom}_{R}(X, Y) \neq 0$. Choose a non-zero homomorphism $\varphi: X \rightarrow Y$ and pick $x \in X$ with $\varphi(x) \neq 0$. By 2.6, we may apply 2.5 which yields an $R$-homogeneous map $f: M \rightarrow M$ such that $f \mid X=\varphi$ and $f(M \backslash X)=0$. Using 1.2 and the independence of $x$ and $f(x)$ it follows that

$$
0=f(f(x)+x)=f\left(f(x)+1_{M}(x)\right)=f\left(f+1_{M}\right)(x)=f f(x)+f(x)=f(x)
$$

which is a contradiction. Conversely, suppose $M$ is absolutely anisotropic. Let $\left\{X_{i}\right\}_{i \in l}$ be the set of all pure rank-one subgroups of $M$. Then $\operatorname{Hom}_{R}\left(X_{i}, X_{j}\right)=0$ whenever $i \neq j$. Let $f \in \mathscr{M}_{R}(M)$. It follows from 3.3 that, for each $i \in I$, $f \mid X_{i} \in \operatorname{Hom}_{R}\left(X_{i}, M\right)$ and, thus, $f \mid X_{i} \in \operatorname{Hom}_{R}\left(X_{i}, X_{i}\right)$. Apply 2.2 .

The torsion-free endomorphal modules over integral domains are precisely those of rank one:

THEOREM 3.5. Let $M$ be a torsion-free module over an integral domain $R$. Then $\mathscr{M}_{R}(M)=E_{R}(M)$ if and only if $M$ has rank one.

PROOF. Assume $M$ is a torsion-free $R$-module of rank at least two. Then there exist two pure rank-one submodules $A$ and $B$ of $M$ such that $A \cap B=0$. Let $X=A \cup B$. By 2.6, $X$ is an $R$-closed and strongly $R$-pure subset of $M$. Apply 2.5 with $\varphi=1_{M} \mid X$ and call the resulting $R$-homogeneous map $f$. Pick $0 \neq a \in A$ and $0 \neq b \in B$. Then

$$
f(a+b)=0 \neq a+b=f(a)+f(b) .
$$

Thus, $M$ is not endomorphal. The proof is completed using 3.3 . 


\section{Modules over Dedekind domains}

Let $R$ be a Dedekind domain. We will give a complete characterization of the semi-endomorphal $R$-modules. Similar to modules over principal ideal domains, modules over Dedekind domains have nice decomposition properties. In particular, every indecomposable module over a Dedekind domain must be either torsion or torsion-free $[9$, p. 336, Theorem 10]. Thus, we have the following consequence of 3.1:

LEMMA 4.1. A semi-endomorphal module over a Dedekind domain is either a torsion module or is torsion-free.

The theory of torsion modules over Dedekind domains is essentially that of torsion modules over principal ideal domains. We summarize results from [9]: Let $\mathscr{P}$ denote the set of all maximal ideals of $R$. For each $P \in \mathscr{P}$ and each $R$-module $M$, let $M_{P}$ denote the set of all elements in $M$ with order ideal a power of $P$. Then $M_{P}$ is a submodule of $M$ and $M_{P}$ can be regarded as a module over the ring $R_{\{P)}=\left\{r s^{-1} \mid r, s \in R, s \notin P\right\}$, the localization of $R$ at $P$. Moreover, $R_{(P)}$ is a discrete valuation domain, in particular a principal ideal domain. If $M$ is torsion then $M=\bigoplus_{P \in \mathscr{P}} M_{P}$

Let $Q$ again denote the quotient field of $R$. Then $Q / R$ is a torsion $R$-module, hence $Q / R=\bigoplus_{P \in \mathscr{P}}(Q / R)_{P}$.

Define $R\left(P^{\infty}\right)=(Q / R)_{P}$. As a factor module of the locally cyclic module $Q$, each $R\left(P^{\infty}\right)$ is locally cyclic; it is also an injective hull of the modules of the form $R / P^{n}$. Each $R / P^{n}$ is a principal ideal ring whose ideal lattice is a chain $[10 ; \mathrm{p} .137,6.20]$. It follows that the submodules of $R\left(P^{\infty}\right)$ are totally ordered. The indecomposable torsion modules are precisely the modules of the form $R / P^{n}$ and $R\left(P^{\infty}\right)$ with $P \in \mathscr{P}$.

As is the case for $\mathbb{Z}$-modules [5, p. 86, Exercise 5], one has

LEMMA 4.2. Every finitely generated submodule of $Q / R$ is cyclic.

A characterization of the endomorphal torsion modules is contained in

THEOREM 4.3. Let $T$ be a torsion module over a Dedekind domain $R$. The following properties are equivalent.

(1) $\mathscr{M}_{R}(T)$ is a ring.

(2) $\mathscr{M}_{R}(T)=E_{R}(T)$.

(3) $T$ is locally cyclic.

(4) $T$ is a submodule of $Q / R$.

ProOF. Let $T=\bigoplus_{P \in \mathscr{P}} T_{P}$ be a torsion module. By 4.2, (4) implies (3), and (3) implies (2), by 2.1. Trivially, (2) implies (1). Assume (1). If each $T_{P}$ is 
indecomposable, $T$ has the desired form. Assume not. Then $T$ contains a direct summand $G=A \oplus B$ with $0 \neq A, B \leq R\left(P^{\infty}\right)$ for some $P$. By 2.3 , the module $G$ must be endomorphal. Let $X=\{a+b \mid a \in A, b \in B, o(a)=o(b)\}$. One verifies that $X$ is an $R$-closed and strongly $R$-pure subset of $G$. Applying 2.5 with $\varphi=1_{G} \mid X$ results in an $R$-homogeneous map $f: G \rightarrow G$. Choose $x \in A$ and $y \in B$ such that $o(x)=o(y)=P$. By 2.4

$$
0 \neq x+y=f(x+y)=f(x)+f(y)=0
$$

which is a contradiction.

Thus, all semi-endomorphal $R$-modules are determined:

COROLLARY 4.4. If $M$ is a module over the Dedekind domain $R$, then $\mathscr{M}_{R}(M)$ is a ring if and only if either (i) $M \leq Q / R$, or (ii) $M$ is torsion-free and absolutely anisotropic.

ProOF. Combine 4.1, 4.3 and 3.4.

COROLlARY 4.5. If $M$ is a module over the Dedekind domain $R$, then $\mathscr{M}_{R}(M)=$ $E_{R}(M)$ if and only if either (i) $M \leq Q / R$, or (ii) $M$ is torsion-free of rank one.

PROOF. Combine 4.1, 4.3 and 3.5.

COROllaRY 4.6. A module $M$ over a Dedekind domain is semi-endomorphal but not endomorphal if and only if $M$ is absolutely anisotropic torsion-free of rank at least two.

If $R$ is a Dedekind domain which has only finitely many maximal ideals, then $R$ is a principal ideal domain [10, p. 144, Exercise 3]. Since $R$ has only finitely many pairwise non-associate primes, there are only finitely many types so that no torsionfree $R$-module of rank two or larger can be absolutely anisotropic. Since, by 3.3, every torsion-free rank-one module must be endomorphal, we have

COROLLARY 4.7. Let $R$ be a Dedekind domain. If there exists a semi-endomorphal $R$-module which is not endomorphal, the prime spectrum of $R$ is infinite.

\section{Modules over principal ideal domains}

Let $R$ be a principal ideal domain. Since $R$ is a Dedekind domain, the characterization problems are solved. Over a principal ideal domain, every torsion-free rank-one module is locally cyclic. In view of $4.5,3.2$ and 2.1 , this implies 
PROPOSITION 5.1. A module over a principal ideal domain is endomorphal if and only if it is locally cyclic.

We turn to Problem 1.5, that is, the existence of semi-endomorphal modules which are not endomorphal. By 4.7, $R$ having a finite prime spectrum implies every semiendomorphal $R$-module is endomorphal. The question remains whether the converse holds.

As remarked earlier, the answer is 'yes' if $R=\mathbb{Z}$. This follows from results of Beaumont and Pierce who posed the problem of finding necessary and sufficient conditions for a given set $\mathbf{T}$ of types to equal the typeset $\mathbf{T}(A)$ of a rank-two torsionfree abelian group $A$ [2]. In our context, we are concerned with the existence of absolutely anisotropic modules, that is, modules whose typesets have the property that any two of its members are incomparable.

For typesets of this sort, the realization problem of [2] has also been considered by Dubois [4], Ito [8], Schultz [17], Arnold and Vinsonhaler [1], and others. Their constructions differ. Given a typeset $\mathbf{T}$, all known constructions of the $\mathbb{Z}$-module $A$ having $\mathbf{T}$ for its typeset involve, at some point, some number theoretical result which is not available for arbitrary principal ideal domains, for example, the prime number theorem: $\lim _{n \rightarrow \infty} n / p_{n}=0$. It is an open question raised in [1] whether a realization theorem can be proved without using some version of this theorem [ibid., p. 20, 5.3].

For our purposes, we are concerned with a much weaker form of the realization problem: all we need is the existence of one typeset $\mathbf{T}$ any two of whose members are incomparable and a torsion-free module $A$ such that $\mathbf{T}=\mathbf{T}(A)$.

Under the special hypothesis that $R$ is a principal ideal domain with sufficiently many primes, this can be proved establishing the existence of absolutely anisotropic torsion-free $R$-modules. The following clever construction was communicated to the authors by Vinsonhaler.

PROPOSITION 5.2. Let $R$ be a principal ideal domain of infinite cardinality $\kappa$. If the set of maximal ideals of $R$ has cardinality $\kappa$, then there exists an absolutely anisotropic torsion-free $R$-module of rank two.

Proof. Assume the hypotheses. Let $Q$ denote the quotient field of $R$ and let $V=Q \oplus Q$ be the two-dimensional vector space over $Q$. Choose an index set $I$ such that $\left\{X_{i}\right\}_{i \in I}$ is the collection of all one-dimensional subspaces of $V$ with $X_{i} \neq X_{j}$ if $i \neq j$. One verifies $|R|=|V|=|I|=\kappa$ so that, by hypothesis, $\mathscr{P}=\left\{P_{i}\right\}_{i \in I}$ with $P_{i} \neq P_{j}$ if $i \neq j$. For each $P_{i} \in \mathscr{P}$, select one prime $p_{i} \in R$ such that $P_{i}=p_{i} R$. Let $A_{i}=\left\{k p_{i}^{-n} \mid k \in R, n \in \mathbb{N}\right\}$. Then $A_{i}$ is an $R$-submodule of $Q$. For each $i \in I$, select a non-zero vector $v_{i} \in X_{i}$ such that $v_{i}=\left(a_{i}, b_{i}\right)$ with $a_{i}$ and $b_{i}$ in $R, a_{i}$ and $b_{i}$ relatively prime. Moreover, if $(1,0) \in X_{j}$, pick $v_{j}=(1,0)$; if $p=p_{i}$ is one of the primes selected earlier and $(p, 1) \in X_{k}$, choose $v_{k}=(p, 1)$. The set 
$v_{i} A_{i}=\left\{v_{i} q \mid q \in A_{i}\right\}$ is an $R$-submodule of $V$. Let $G=\sum_{i \in l} v_{i} A_{i}$. If $x \in G$ is a non-zero element, then $x Q=X_{i}$ for some $i$, and the pure submodule $\langle x\rangle_{*}=G \cap X_{i}$ of $G$ generated by $x$ contains $v_{i} A_{i}$. Hence, $x$ is divisible in $G$ by every power of $p_{i}$. Assume, by way of contradiction, $G$ is not absolutely anisotropic. Then there exist two independent elements $x$ and $y$ in $G$ whose types are comparable. It follows that there is a prime $p \in R$ such that $G$ is $p$-divisible. Let $j \in I$ such that $p=p_{j}$. Choose $k \in I$ different from $j$ and let $v=v_{k}$. Since $G=G p$, there is $x \in G$ such that $v=x p$ and $x=\sum_{i \in F} v_{i} k_{i} p_{i}^{-m_{i}}$ where $F \subset I$ is finite, $k_{i} \in R$, the $m_{i}$ are non-negative integers, and $m_{i}>0$ implies $p_{i}$ is no divisor of $k_{i}$. We claim that $j \in F$ and $m_{j}=1$. Indeed, if $r=\prod_{i \in F} p_{i}^{m_{i}}$ and $r_{i}=r p_{i}^{-m_{i}}$, we have $v r=\sum_{i \in F} v_{i} k_{i} r_{i} p$, and the coordinates of $v$ being relatively prime implies $j \in F$ and $m_{j} \geq 1$. Hence $k_{j}$ is not divisible by $p=p_{j}$, so that because of $v_{j} k_{j} r_{j} p=v r-\sum_{i \neq j} v_{i} k_{i} r_{i} p \in(R \oplus R) p^{m_{j}}$ the coordinates of $v_{j}$ both are divisible by $p^{m_{j}-1}$. Thus, $m_{j}=1$ and $r=p r_{j}$. This implies $\left(v-v_{j} k_{j}\right) r_{j} \in(R \oplus R) p$ so that

$$
v-v_{j} k_{j} \in(R \oplus R) p .
$$

Recall that $v=v_{k}$ where $k \neq j$ was arbitrary. For the final contradiction we distinguish two cases: if $p_{j}$ divides $a_{j}$, then $v_{j} \neq(1,0)$ so that $(1,0)=v_{k}$ for some $k \neq j$ in $I$; if $p_{j} \nmid a_{j}$ then $v_{j} \neq(p, 1)$ and we have $(p, 1)=v_{k}$ for some $k \neq j$. In either case, $v=v_{k}$ cannot satisfy (5.3). This completes the proof.

COROLLARY 5.3. Let $R$ be a principal ideal domain whose prime spectrum has the same cardinality as $R$. Then there exists an $R$-module $M$ which is semi-endomorphal but not endomorphal.

ProOF. Observe 4.6.

\section{Acknowledgement}

The authors would like to express their appreciation to C. J. Maxson and A. P. J. van der Walt for graciously sharing preprints of their work.

\section{References}

[1] D. M. Amold and C. I. Vinsonhaler, 'Typesets and cotypesets of rank-2 torsion free abelian groups', Pacific J. Math. 114 (1984), 1-21.

[2] R. A. Beaumont and R. S. Pierce, Torsion free groups of rank two, Mem. Amer. Math. Soc. (Amer. Math. Math. Soc., Providence, 1961). 
[3] H. Cartan and S. Eilenberg, Homological algebra (Princeton University Press, Princeton, 1956).

[4] D. Dubois, 'Applications of analytic number theory to the study of typesets of torsion free abelian groups I', Publ. Math. 12 (1965), 59-63.

[5] L. Fuchs, Infinite Abelian groups, Vol. I, (Academic Press, New York, 1970).

[6] _ Infinite Abelian groups, Vol. II, (Academic Press, New York, 1973).

[7] P. Fuchs, C. J. Maxson and G. Pilz, 'On rings for which homogeneous maps are linear', Proc. Amer. Math. Soc. 112 (1991), 1-7.

[8] R. Ito, 'On type-sets of torsion free abelian groups of rank two', Proc. Amer. Math. Soc. 48 (1975), $39-42$.

[9] I. Kaplansky, 'Modules over Dedekind rings and valuation rings', Trans. Amer. Math. Soc. 72 (1952), 327-340.

[10] M. D. Larsen and P. J. McCarthy, Multiplicative ideal theory (Academic Press, New York, 1971).

[11] C. J. Maxson and K. C. Smith, 'Simple near-ring centralizers of finite rings', Proc. Amer. Math. Soc. 75 (1979), 8-12.

[12] —_- 'Near-ring centralizers', in: Proceedings Ninth Annual USL Mathematics Conference Research Series 48 (Univ. Southwestern Louisiana, 1979) pp. 49-58.

[13] —_. 'Centralizer near-rings that are endomorphism rings', Proc. Amer. Math. Soc. 80 (1980), 189-195.

[14] C. J. Maxson and A. P. J. van der Walt, 'Centralizer near-rings over free ring modules', J. Austral. Math. Soc. 50 (1991), 279-296.

[15] — , 'Homogeneous maps as piecewise endomorphisms', Comm. Algebra 20 (1992), 27552776.

[16] G. Pilz, Near-rings, 2nd edition (North Holland, Amsterdam, 1983).

[17] P. Schultz, 'The typeset and cotypeset of a rank two abelian group', Pacific J. Math. 70 (1978), 503-517.

[18] D. W. Sharpe and P. Vámos, Injective modules (Cambridge University Press, Cambridge, 1972).

\section{University of Houston}

Houston

Texas 77204-3476

e-mail addresses: hausen@uh.edu, jjohnson@uh.edu 